\title{
Diversité des insectes actifs au sol dans quatre écosystèmes de bas-fonds du Burkina Faso : importance pour la détermination de bio-indicateurs caractérisant ces milieux
}

\author{
E. ILBOUDO-TAPSOBA ${ }^{1}$, H. TANKOANO ${ }^{2}$, M. OUEDRAOGO ${ }^{3}$, I. O. DICKO ${ }^{2}$ et \\ A. SANON ${ }^{1 *}$ \\ ${ }^{1}$ Laboratoire d'Entomologie Fondamentale et Appliquée, UFR/SVT, Université de Ouagadougou, 06 BP 9499 \\ Ouagadougou, Burkina Faso, \\ ${ }^{2}$ Institut du Développement Rural, Université Polytechnique de Bobo-Dioulasso, Burkina Faso. \\ ${ }^{3}$ Musée d'Histoire Naturelle (IRBET), Centre National de la Recherche Scientifique et Technologique \\ (CNRST), Burkina Faso. \\ *Auteur correspondant, E-mail: sanonant@yahoo.fr
}

\section{RESUME}

L'action de l'Homme sur l'environnement peut affecter la diversité biologique qui à son tour peut être utilisée comme indicateur de la santé des écosystèmes. Une étude a été menée en 2006 dans quatre (4) Micro Bassin-Versants (MBV) du Burkina Faso pour évaluer la diversité des insectes rampants au sol et caractériser chaque écosystème par ses bio-indicateurs les plus significatifs. La diversité taxonomique des espèces d'insectes capturées dans différents sites par des pièges de Barber, leur distribution et leur abondance relative au début et à la fin de la saison des pluies ont ainsi été déterminées. Quatre (4) ordres regroupant treize (13) familles d'insectes, les Carabidae, les Scarabaeidae, les Tenebrionidae, les Cétonidae, les Cérambycidae, les Curculionidae, les Hydrophilidae, les Elateridae, les Staphylinidae, les Chrysomelidae (Coléoptères), les Formicidae (Hyménoptères), les Gryllidae (Orthoptères) et les Labiduridae (Dermaptères), ont été identifiées dans les MBV étudiés. Les variations climatiques influencent la diversité taxonomique des insectes qui fluctue par ailleurs dans l'espace et dans le temps. Cinq (5) familles, les Formicidae, les Cicindellidae, les Carabidae, les Scarabaeidae et les Tenebrionidae ont été les plus abondantes et les plus diversifiées en espèces dans chacun des quatre MBV. Ces taxa, qui se sont adaptés aux écosystèmes étudiés, en sont probablement les meilleurs indicateurs. Les résultats obtenus sont discutés en vue de déterminer comment ces groupes majeurs peuvent être utilisés dans l'évaluation et la gestion des écosystèmes qu'ils peuplent.

(C) 2011 International Formulae Group. All rights reserved.

Mots-clés : Diversité biologique, Bio-indicateurs, Insectes, Gestion de l'environnement, Ecosystèmes marginaux.

\section{INTRODUCTION}

Les effets directs et indirects de l'activité humaine sur les systèmes écologiques et leur biodiversité constituent une préoccupation mondiale majeure (Green et al., 2005). Cela est d'autant plus que la transformation et la fragmentation des habitats naturels par l'Homme, leurs actions négatives sur la nature comme les feux de brousse, la coupe abusive de bois, la divagation des 
animaux, entraînent une érosion et une perte de la biodiversité (Groombridge and Jenkins, 2002 ; Powledge, 2002 ; Green et al., 2005). Nous nous situons aujourd'hui dans une crise économique et environnementale mondiale, de plus en plus exacerbée dans les pays tropicaux, particulièrement pauvres, où la pression anthropique sur les ressources naturelles est très importante. En effet, le Burkina Faso en est un exemple illustratif. La surexploitation des terres sans amendement conséquent ainsi que l'érosion (hydrique et éolienne) appauvrissent les sols. La croissance démographique galopante et la baisse de la pluviosité poussent les jeunes à migrer vers des terres encore vierges (Mathieu, 1994). Les terres marginales sont ainsi colonisées, mais c'est surtout les bas-fonds et les zones sud soudaniennes qui sont recherchés par les migrants agricoles. De ce fait, les paysages anthropiques gagnent rapidement sur les milieux naturels (environ $60 \%$ du territoire en 2000), au détriment des équilibres écologiques et de la biodiversité (Augusseau et al., 2000). Le contexte de sécheresses récurrentes, de baisse continue et de variabilité de la pluviométrie que connaît le pays fait que les bassins versants sont particulièrement prisés par les communautés rurales, y compris les agriculteurs autochtones, les migrants agricoles et les éleveurs sédentaires et transhumants. De ce fait, certaines ressources deviennent facilement des pôles de dégradation environnementale ou des zones de conflits. Aussi, une bonne gestion des ressources des différents écosystèmes nécessite une évaluation efficace et régulière de l'impact des différentes interventions humaines ou autres sur ces milieux.

La présente étude veut contribuer à cela en s'appuyant sur l'évaluation de la diversité des Arthropodes, particulièrement les Insectes, comme un indicateur de la santé des milieux à un moment donné, ou un indicateur de son évolution au bout d'un certain temps. Contrairement à l'idée bien répandue selon laquelle les insectes sont surtout nuisibles (Samways and Lockwood, 1998 ; Lawton, 2001), ils peuvent aussi être très utiles à l'homme, intervenant à tous les niveaux trophiques. Certains auteurs estiment même que les écosystèmes seraient inopérants sans la présence des insectes (Wiggins et al., 1991). En effet, leur importance écologique prépondérante les rend utiles pour l'évaluation des perturbations ou des incidences sur l'environnement de diverses sortes (Duelli and Obrist, 2003). De façon plus précise, les insectes peuvent être des indicateurs sensibles de l'intégrité écologique d'un écosystème, du maintien de sa productivité, de sa stabilité ou de sa résilience (New, 1999). De ce point de vue, nous pensons comme plusieurs auteurs qu'une étude basée uniquement sur les gros animaux, bien que plus facile, ne donnera qu'un aperçu biaisé de la dynamique réelle des écosystèmes (Kremen et al., 1993; Finnamore, 1996). Au cours de cette étude, nous avons caractérisé les écosystèmes de bas-fonds de quatre (4) Micro Bassin-Versants (MBV) du Soum, du Sanmatenga, du kouritenga et de la Kompienga par leurs peuplements d'insectes échantillonnés avec des pièges de Barber, efficaces pour capturer les Arthropodes rampants au sol. Ce biotope peut effectivement abriter de nombreux groupes d'Arthropodes participant à différents processus écologiques importants comme la prédation, le recyclage de la matière organique, l'ameublissement du sol, le nettoyage des déchets. Les résultats obtenus permettront de comparer les différents milieux étudiés quant à leur diversité en insectes bioindicateurs.

\section{MATERIEL ET METHODES \\ Caractéristiques physiques des Micro Bassin-versants (MBV) \\ Le MBV de la province du Soum}

Le Micro Bassin-Versant (MBV) du Béli, dans la province du Soum au Nord du Burkina Faso est compris entre les parallèles $13^{\circ} 51^{\prime} 10,8^{\prime}$ ' et $14^{\circ} 40^{\prime} 40,8^{\prime}$ ' de Latitude Nord et entre les méridiens $1^{\circ} 16^{\prime} 30^{\prime}$ ' et $2^{\circ} 03^{\prime} 39,6^{\prime}$ ', de Longitude Ouest couvrant une superficie d'environ 34201 ha (Silem, 2006a). Le climat est du type sahélien caractérisé par une saison pluvieuse s'étalant de Juin à Septembre avec 
des hauteurs annuelles moyennes de pluies inférieures à $600 \mathrm{~mm}$, et une saison sèche le reste de l'année. La végétation, décrite par Fontès and Guinko (1995), est actuellement abondamment dégradée en raison de la détérioration des conditions climatiques et des actions anthropiques. Elle est caractérisée par une strate herbacée, dont les principales espèces végétales de la famille des Poaceae sont Cenchrus biflorus, Aristida mutabilis, Schoenefeldia gracili et Schoenefeldia gracilis dans les bas-fonds, les mares et les dépressions ouvertes. Cette strate herbacée est associée à une strate arbustive basse et claire dont les espèces dominantes sont Combretum glutinosum (Combretaceae), Guiera senegalensis (Combretaceae), Acacia senegal (Leguminosae-Mimosaideae) et Acacia raddiana (Mimosaceae). Les sols les plus représentés au niveau du MBV sont des sols peu lessivés.

\section{Le MBV du Sanmatenga}

Le Micro Bassin-Versant de Korsimoro, couvert par l'étude, s'étend dans les provinces du Sanmatenga et du Namentenga et appartient au bassin versant du Nakambé. Son exutoire peut se repérer selon les coordonnées $01^{\circ} 04$ ' de Longitude Ouest et $12^{\circ} 49^{\prime}$ de Latitude Nord. Sa superficie totale est de 107949 ha dont 95,3\% sont situés dans le Sanmatenga (Silem, 2006b). Il est localisé dans la zone climatique Nord-Soudanienne (Fontès and Guinko, 1995), caractérisée par une saison hivernale de Juin à Septembre. Le MBV de Korsimoro est situé entre les isohyètes $600 \mathrm{~mm}$ et $800 \mathrm{~mm}$. La végétation $\mathrm{du}$ bassin versant comprend une savane arbustive dominée par Combretun micranthum, Combretum glutinosum, Combretum nigricans, Anogeissus leiocarpus (Combretaceae), une savane arborée dominée par Balanites aegyptiaca (Mimosaceae), Lannea microcarpa (Anacardiaceae) et Acacia spp. (Mimosaceae), des forêts galeries le long des cours d'eau dominées par Mitragyna inermis (Rubiaceae), Anogeissus leiocarpus (Combretaceae) et Combretum micranthum (Combretaceae), des steppes arbustives et des steppes arborées. Les sols sont en général pauvres en matières organiques et en argile.

\section{Le MBV du Kouritenga}

Dénommé Koulouko, ce MBV s'étend également dans les provinces voisines du Namentenga, du Ganzourgou et de la Gnagna. Il est situé sur une pénéplaine avec un relief généralement plat. Cependant on note la présence de quelques monticules par endroits, de quelques affleurements granitiques et de la cuirasse latéritique (Silem, 2005). D'un point de vue climatique, le MBV se situe dans le secteur Nord soudanien (Fontès and Guinko, 1995). Les précipitations annuelles vont de $750 \mathrm{~mm}$ à $1000 \mathrm{~mm}$. La saison sèche dure 6 à 7 mois. La végétation présente des savanes ayant partout l'allure de paysages agraires dominés ça et là par de gros arbres trapus de 10 à $20 \mathrm{~m}$ de hauteur appartenant aux espèces intégralement protégées telles Acacia albida (Leguminosae-Mimosaideae), Adansonia digitata (Bombacaceae), Vitellaria paradoxa (Sapotaceae), Lannea microcarpa (Anacardiaceae), Parkia biglobosa (Leguminosae-Mimosioideae), Tamarindus indica (Leguminosae-Caesalpinioideae). Les jachères récentes et les sols fortement érodés sont colonisés par de nombreuses espèces sahéliennes. Les sols présentent une grande variabilité morphologique allant des sols tropicaux ferrugineux, aux sols ferrugineux tropicaux lessivés et aux sols ferrugineux tropicaux lessivés à concrétions ou hydromorphes.

\section{Le MBV de la Kompienga}

Il est situé dans la région Est du Burkina, à cheval sur les deux départements de Pama et de Kompienga, couvrant une superficie de 93 001,3 ha. Le MBV est situé entre $0^{\circ} 30^{\prime}$ et $0^{\circ} 40^{\prime}$ de Longitude Est et entre $11^{\circ} 05^{\prime}$ et $11^{\circ} 20^{\prime}$ de Latitude Nord (Silem, 2006c).

Le climat est caractérisé par une pluviométrie de 900 à $1000 \mathrm{~mm}$ par an, en une saison hivernale qui dure de quatre à cinq mois à partir de mai-juin. La végétation du micro bassin est comprise dans le secteur phytogéographique soudanien septentrional selon le découpage de Fontès and Guinko 
(1995). Elle est cependant fortement marquée par les empreintes d'une pression anthropique récente. La végétation présente des savanes arbustives claires dominées par Combretum glutinosum, Combretum ghazalense (Combretaceae), Balanites aegyptiaca (Balanitaceae), Acacia sp (LeguminosaeMimosioideae), Piliostigma spp (Leguminosae-Caesalpinioideae), Vitellaria paradoxa (Sapotaceae), des Savanes arborées clairsemées dominées de Terminalia avicenniö̈des (Combretaceae), Anogeissus leocarpus (Combretaceae) , Diospyros mespiliformis (Ebenaceae), Tamarindus indica (Leguminosae-Caesalpinioideae), Vitelleria paradoxa, (Sapotaceae), Parkia biglobosa (Leguminosae-Mimosioideae), Lannea microcarpa (Anacardiaceae), Adansonia digitata (Bombacaceae), Mytragina inermis (Rubiaceae), de tapis herbacé dense caractérisé par des espèces pérennes telles Andropogon gayanus, Pennisetum pedicellatum, Brachiaria jubata. (Poaceae). Les sols sont à dominance ferrugineux tropicaux lessivés de profondeur variable avec des vertisols et des sols hydromorphes à Gley et pseudo Gley.

\section{Méthode de collecte des données \\ Choix des sites d'étude}

A l'aide d'une carte hydrographique de chaque province, les principaux bassins versants ont été examinés et lors d'une prospection sur le terrain, des sites ont été retenus dans chaque Micro Bassin-Versant (MBV) en tenant compte des variabilités écologiques et des effets des actions anthropiques (coupes de bois, présence de champs etc.). Les sites étaient localisés, dans la mesure du possible, en amont, en zone intermédiaire et en aval des bassins versants (Figure 1). L'ensemble des sites retenus figure dans le Tableau 1. Au total onze (11) sites (Kaboanga II, Toutourgou, Oumpougdéni, Nabnongzougou, Kampelcezougou, Nakambé, Koutoumtenga, Damba, Sê, Fili -fili, Bani) ont été retenus. Dans chaque bassin versant, trois transects principaux ont été installés : un transect en amont, un transect dans la zone intermédiaire et un transect en aval. Les transects ont été orientés perpendiculairement au cours d'eau principal. A chaque transect principal étaient associés trois transects secondaires. La distance entre deux transects est de 50 à $100 \mathrm{~m}$.

\section{La collecte des insectes}

Sur chaque transect au moins deux (2) placettes de $50 \mathrm{~m}$ x $20 \mathrm{~m}\left(1000 \mathrm{~m}^{2}\right)$ ont été délimitées par des bornes. Les insectes rampants au sol étaient échantillonnés au cours de deux périodes, en début de saison pluvieuse (DSP), de juin à juillet et en fin de saison pluvieuse (FSP) de septembre à octobre en vue de déterminer l'évolution de leur diversité et de leur abondance en fonction des saisons. Pour cela, dans chaque placette étaient implantées cinq (5) boîtes cylindriques d'1 litre de contenance utilisées comme pièges de Barber et disposées aux quatre (4) angles puis au milieu de la placette. De l'alcool à 40 ${ }^{\circ} \mathrm{C}$ était introduit dans les boîtes jusqu'au tiers inférieur pour immobiliser les insectes qui y tombaient. Vingt quatre heures $(24 \mathrm{~h})$ après leur implantation, les boîtes étaient récupérées par placette et leur contenu était transvasé dans des bocaux contenant de l'alcool à $70{ }^{\circ} \mathrm{C}$ avant d'être ramené au laboratoire pour le tri et l'identification jusqu'au niveau spécifique lorsque cela était possible.

L'identification des spécimens et détermination d'indices écologiques

Tous les spécimens ont pu être identifiés au moins jusqu'au genre à l'aide de différentes clés de détermination (Delobel and Tran, 1993 ; Delvare and Aberlenc, 1999), mais aussi grâce à des spécimens de référence au Muséum de l'International Institute of Tropical Agriculture (IITA) basé à Cotonou (Bénin) et au Laboratoire des Invertébrés Terrestres de l'Institut Fondamental d'Afrique Noire (IFAN) basé à Dakar au Sénégal.

Les données recueillies ont été utilisées pour calculer des indices écologiques classiques qui permettent d'étudier la structure 


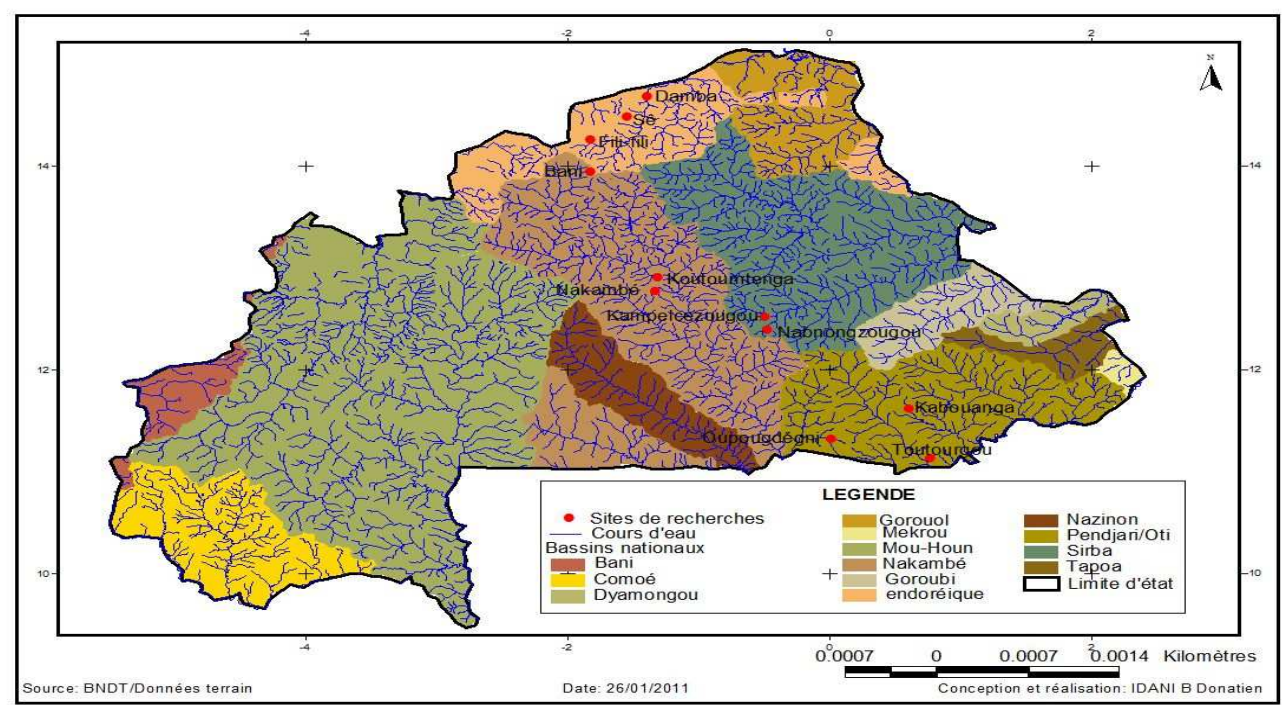

Figure 1 : Carte de localisation des sites et des Micro Bassin-versants étudiés au Burkina Faso en 2006.

Tableau 1 : Identification et caractéristiques des sites d'études dans les quatre (4) Micro Bassin-Versants (MBV) du Burkina Faso en 2006.

\begin{tabular}{ccccccc}
\hline \multirow{2}{*}{ MBV } & \multicolumn{2}{c}{ Amont } & \multicolumn{2}{c}{ Zone intermédiaire } & \multicolumn{2}{c}{ Aval } \\
\cline { 2 - 7 } Soum & $\mathbf{A +}$ & $\mathbf{A + +}$ & $\mathbf{A +}$ & $\mathbf{A + +}$ & $\mathbf{A +}$ & $\mathbf{A + +}$ \\
Sanmatenga & - & Damba & - & Sê & Bani & Fili-Fili \\
Kouritenga & - & Nabnongzougou & Kampelcezougou & - & - & - \\
Kompienga & Kaboanga II & - & - & Koutoumtenga & - & - \\
A+ : site moyennement anthropisé $;$ A++ : site fortement anthropisé $;$ \\
Sites identifiés : Kaboanga II, Toutourgou, Oumpougdéni, Nabnongzougou, Kampelcezougou, Nakambé, Koutoumtenga, Damba, Sê, Fili -fili, Bani.
\end{tabular}


des peuplements et d'évaluer leur biodiversité en fonction de l'espace et de la période de récolte. Les indices calculés sont :

- la richesse spécifique (Magurran, 2004) des insectes, déterminée par le nombre total d'espèces répertoriées dans chaque Micro Bassin-Versant par saison.

- l'indice de diversité de SHANNONWIENER, H' (Magurran, 2004) exprime la diversité du peuplement. Il est déterminé à partir du nombre d'individus par espèce et par province en fonction de la saison par la formule $\mathrm{H}^{\prime}=-\Sigma$ ((qi/Q) $\left.\log _{2}(\mathrm{qi} / \mathrm{Q})\right)$. où qi représente le nombre d'individus du taxon $\mathrm{i}$ et $\mathrm{Q}$ est le nombre total d'individus dans le peuplement.

La diversité est maximale lorsque tous les taxons observés ont la même abondance. $\mathrm{H}^{\prime} \max =\log _{2} \mathrm{~S} ; \mathrm{S}$ est le nombre total de taxons dans le peuplement.

- l'indice d'équitabilité de PIELOU J' (Magurran, 2004) évalue l'équirépartition du peuplement. Il permet de définir la régularité qui est la diversité observée rapportée à la diversité maximale et de comparer des écosystèmes très différents par leur richesse spécifique. Il donne ainsi une idée sur la qualité de la structuration du peuplement. Il est calculé par la formule : J' = H'/H' max.

\section{Analyses statistiques}

Les indices de diversité et leurs variations dans l'espace et dans le temps ont été comparés dans différents milieux grâce à une Analyse de variance (ANOVA) de Kruskal-Wallis en utilisant le logiciel Statistica version 7.1. Le test de Kruskal Wallis est un test non paramétrique indiqué pour l'analyse d'échantillons indépendants dont la normalité n'est pas prouvée (Ruxton and Beauchamp, 2008). Le seuil de signification retenu est de $5 \%$.

\section{RESULTATS}

Résultats globaux

Les insectes collectés au cours de l'étude appartiennent principalement à quatre
(4) ordres : Coléoptères, Hyménoptères, Orthoptères et Dermaptères. Les familles dominantes ont été respectivement les Carabidae (20 espèces), les Formicidae (11 espèces), les Scarabaeidae (10 espèces), les Tenebrionidae ( 9 espèces) et les Cicindellidae (5 espèces). Les coléoptères avec dix (10) familles recensées sont les plus représentatifs (Tableau 2). La richesse spécifique totale est alors estimée à 64 espèces (Tableau 2).

La diversité de ces familles varie en fonction de la période d'échantillonnage et du Micro Bassin-Versant (MBV) d'étude (Tableau 3). Ainsi la diversité des Carabidae était plus importante à la Kompienga, au Sanmatenga et au Soum comparativement au Kouritenga. Une plus grande diversité de Scarabaeidae et de Cicindellidae a été obtenue au Soum. Un nombre presque équivalent d'espèces de Formicidae (9 à 11 espèces) a été observé dans les 4 provinces. Une plus faible diversité de Tenebrionidae (2 espèces) a été observée dans le MBV du Sanmatenga en comparaison aux autres MBV (6-8 espèces).

\section{Evolution des indices de diversité entomologique dans l'espace et dans le temps}

Les différents indices déterminés par province et par période d'étude sont présentés dans le Tableau 4. La richesse spécifique apparaît plus élevée au Soum comparativement à celle de Kompienga, de Sanmatenga et de Kouritenga. Cependant, l'indice de diversité de Shannon reste faible et inférieur à la diversité théorique calculée dans toutes les quatre (4) provinces, indépendamment de la période d'échantillonnage. Les valeurs de J'déterminant la régularité s'écartent toujours de la valeur 1 , et se situent très souvent en deçà de 0,5. Il n'y a donc pas une équirépartition des peuplements au sein de tous les milieux étudiés. Néanmoins, la régularité semble s'améliorer à la fin de la saison pluvieuse. 
E. ILBOUDO -TAPSOBA et al. / Int. J. Biol. Chem. Sci. 5(2): 724-738, 2011

Tableau 2 : Richesse spécifique des familles d'insectes de différents ordres collectées dans les quatre (4) Micro Bassin-Versants (MBV). Burkina Faso, 2006

\begin{tabular}{llc}
\hline Ordres & Familles & Nombre d'espèces \\
\hline & Carabidae & 20 \\
& Scarabaeidae & 10 \\
& Tenebrionidae & 9 \\
& Cicindellidae & 5 \\
Coléoptères & Cerambycidae & 1 \\
& Curculionidae & 1 \\
& Hydrophilidae & 2 \\
& Elateridae & 1 \\
Hyménoptères & Staphilinidae & 1 \\
Orthoptères & Chrysomelidae & 1 \\
Dermaptères & Formicidae & 11 \\
Richesse totale & Gryllidae & 1 \\
& Labiduridae & 1 \\
\hline
\end{tabular}


Tableau 3 : Espèces d'insectes identifiées à chaque période d'échantillonnage dans les quatre (4) Micro Bassin-Versants (MBV) d'études. Burkina Faso, 2006.

\begin{tabular}{|c|c|c|c|c|c|c|c|c|c|}
\hline \multirow[b]{3}{*}{ Famille } & \multirow[b]{3}{*}{ Nom scientifique } & \multicolumn{8}{|c|}{ Provinces } \\
\hline & & \multicolumn{2}{|c|}{ Kompienga } & \multicolumn{2}{|c|}{ Kouritenga } & \multicolumn{2}{|c|}{ Sanmatenga } & \multicolumn{2}{|c|}{ Soum } \\
\hline & & $D S P$ & FSP & $D S P$ & FSP & $D S P$ & $\overrightarrow{F S P}$ & $D S P$ & FSP \\
\hline \multirow[t]{21}{*}{ Carabidae } & Chlaenius (Chlaenites) columbinus (Dejean) & $\mathrm{x}$ & $\mathrm{x}$ & $\mathrm{X}$ & & $\mathrm{x}$ & $\mathrm{x}$ & $\mathrm{x}$ & \\
\hline & Chlaenius (Chlaenites) senegalensis (Dejean) & $\mathrm{x}$ & & & & $\mathrm{x}$ & $\mathrm{x}$ & $\mathrm{x}$ & $\mathrm{x}$ \\
\hline & Chlaenius (Chlaenites) sp .1 & $\mathrm{x}$ & $\mathrm{x}$ & $\mathrm{x}$ & $\mathrm{x}$ & $\mathrm{x}$ & $\mathrm{x}$ & $\mathrm{x}$ & $\mathrm{x}$ \\
\hline & Chlaenius (Chlaenites) sp.2 & $\mathrm{x}$ & $\mathrm{x}$ & $\mathrm{x}$ & $\mathrm{x}$ & & & $\mathrm{x}$ & $\mathrm{x}$ \\
\hline & Chlaenius (Spilochlaenites) crutiatis (Dejean) & & & & & & & & $\mathrm{x}$ \\
\hline & Chlaenius (Spilochlaenites) boisduvali (Dejean) & & & & & $\mathrm{x}$ & & $\mathrm{x}$ & \\
\hline & Pachydinodes conformis (Dejean) & & & & & & $\mathrm{x}$ & $\mathrm{x}$ & $\mathrm{x}$ \\
\hline & Systocranius senegalensis & $\mathrm{x}$ & & & & & & & \\
\hline & Galeritola africana (Dejean) & $\mathrm{x}$ & $\mathrm{x}$ & & & $\mathrm{x}$ & $\mathrm{x}$ & & \\
\hline & Lonchosternus sp. & $\mathrm{x}$ & $\mathrm{x}$ & & & & $\mathrm{x}$ & & \\
\hline & Siagona sp. & & & & & & $\mathrm{x}$ & & \\
\hline & Calosoma (Stenosta) senegalensis (Dejean) & & $\mathrm{x}$ & & & & & & $\mathrm{x}$ \\
\hline & Tomochilus carbonatus (Chaudoir) & $\mathrm{x}$ & $\mathrm{x}$ & & & $\mathrm{x}$ & $\mathrm{X}$ & & \\
\hline & Neochryopus savage (Hope) & $\mathrm{x}$ & $\mathrm{x}$ & & & & & $\mathrm{x}$ & $\mathrm{x}$ \\
\hline & Tetragonoderus ouadrum (Fabricius) & & & & & & $\mathrm{x}$ & & $\mathrm{x}$ \\
\hline & Brachinus sp.1 & & & & & & $\mathrm{x}$ & $\mathrm{x}$ & $\mathrm{x}$ \\
\hline & Brachinus sp.2 & $\mathrm{x}$ & $\mathrm{x}$ & & & & $\mathrm{x}$ & $\mathrm{x}$ & \\
\hline & Drypta ruficollis (Dejean) & $\mathrm{x}$ & & & & $\mathrm{x}$ & & & \\
\hline & Epomis croesus (Fabricius) & $\mathrm{x}$ & $\mathrm{x}$ & & & & & $\mathrm{x}$ & $\mathrm{x}$ \\
\hline & Callistö̈des $s p$. & $\mathrm{x}$ & & $\mathrm{x}$ & & & & & \\
\hline & Total Carabidae par MBV & & & & & & & & \\
\hline \multirow[t]{7}{*}{ Scarabaeidae } & Gymnopleurus fulgidus (Olivier) & $\mathrm{x}$ & & & & $\mathrm{x}$ & & $\mathrm{x}$ & $\mathrm{x}$ \\
\hline & Gymnopleurus sp. & $\mathrm{x}$ & & & & $\mathrm{x}$ & & $\mathrm{x}$ & \\
\hline & Anomala denuda (Arrow) & & & & & $\mathrm{x}$ & & $\mathrm{x}$ & \\
\hline & Allogymnopleurus umbrinus (Gersteacker) & $\mathrm{x}$ & & $\mathrm{x}$ & & $\mathrm{x}$ & & $\mathrm{x}$ & \\
\hline & Catharsius sp. 1 & & $\mathrm{x}$ & $\mathrm{x}$ & & $\mathrm{x}$ & & $\mathrm{x}$ & $\mathrm{x}$ \\
\hline & Catharsius sp.2 & & $\mathrm{x}$ & $\mathrm{x}$ & & $\mathrm{x}$ & & $\mathrm{x}$ & $\mathrm{x}$ \\
\hline & Copris sp. & & & & & & & $\mathrm{x}$ & \\
\hline
\end{tabular}


E. ILBOUDO -TAPSOBA et al. / Int. J. Biol. Chem. Sci. 5(2): 724-738, 2011

Onthophagus obliquus (Olivier)

Bolboceras sp.

Gametis sanguinolenta (Olivier)

Total Scarabaeidae par MBV

Tenebrionidae

Gonocephalum sculptithorax (Gridelli)

Pimelia senegalensis(Olivier)

Pimelia grandis latastei (Senac)

Thalpophilodes schweinfurthi carinifrons (Fairmaire)

Vieta sp.

Adesmia (Macropoda) variolis (Olivier)

Zophosis quadrilineata (Olivier)

Gonecnemis sp.

Pachycera tagenioides (Eschsholtz)

Total Tenebrionidae par MBV

Cicindellidae Megacephala megacephala(Olivier)

Megacephala ouadrisignata (Dejean)

Cicindela neglecta (Dejean)

Cicindela (Myriochile) Dorsata (Brullé)

Cicindela sp.

Total Cicindellidae par MBV

Formicidae

Cataglyphis congolensis (Stitz)

Pachycondyla analis (Latreille)

Pachycondyla (Brachyponera) sp.

Camponatus maculatus (Fabricius)

Camponatus sericeus (Forel)

Dorylus fulvus (Schuck)

Messor galla (Mayr)

Tetramorium sp.

Monomorium bicolor (Emery)

Monomorium sp.

Pheidole megacephala (Fabricius)

Total Formicidae par MBV

\begin{tabular}{|c|c|c|c|c|c|c|c|c|}
\hline & & & & & & & $\begin{array}{l}\mathrm{X} \\
\mathrm{x}\end{array}$ & $\mathrm{x}$ \\
\hline & \multicolumn{2}{|c|}{5} & \multicolumn{2}{|c|}{3} & \multicolumn{2}{|c|}{6} & \multicolumn{2}{|c|}{9} \\
\hline & $\mathrm{X}$ & $\mathrm{X}$ & $\mathrm{X}$ & $\mathrm{X}$ & $\mathrm{x}$ & $\mathrm{X}$ & $\mathrm{X}$ & $\mathrm{x}$ \\
\hline & & $\mathrm{x}$ & & & & $\mathrm{x}$ & $\mathrm{x}$ & $\mathrm{x}$ \\
\hline & & & & & & & $\mathrm{x}$ & $\mathrm{x}$ \\
\hline \multirow[t]{25}{*}{$s$ (Fairmaire) } & & $\mathrm{X}$ & & & & & $\mathrm{x}$ & $\mathrm{x}$ \\
\hline & $\mathrm{X}$ & $\mathrm{x}$ & & $\mathrm{x}$ & & & & \\
\hline & & & $\mathrm{x}$ & & & & $\mathrm{X}$ & \\
\hline & $\mathrm{X}$ & $\mathrm{X}$ & $\mathrm{x}$ & $\mathrm{X}$ & & & $\mathrm{x}$ & $\mathrm{x}$ \\
\hline & $\mathrm{X}$ & & & $\mathrm{X}$ & & & $\mathrm{X}$ & \\
\hline & $\mathrm{X}$ & $\mathrm{X}$ & & $\mathrm{X}$ & & & $\mathrm{X}$ & $\mathrm{x}$ \\
\hline & & & & & & & & \\
\hline & & $\mathrm{X}$ & $\mathrm{x}$ & & & & $\mathrm{X}$ & \\
\hline & & & & & & & & $\mathrm{x}$ \\
\hline & $\mathrm{X}$ & & $\mathrm{x}$ & & & & $\mathrm{X}$ & \\
\hline & & & & & & & $\mathrm{X}$ & \\
\hline & & & & & & & $\mathrm{x}$ & \\
\hline & & & & & & & & \\
\hline & & & & $\mathrm{x}$ & $\mathrm{x}$ & & $\mathrm{x}$ & $x$ \\
\hline & $\mathrm{X}$ & $\mathrm{X}$ & 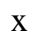 & $\mathrm{X}$ & $\mathrm{X}$ & $\mathrm{X}$ & $\mathrm{x}$ & $x$ \\
\hline & $\mathrm{x}$ & $\mathrm{x}$ & $\mathrm{x}$ & $\mathrm{x}$ & $\mathrm{x}$ & $\mathrm{X}$ & $\mathrm{x}$ & $\mathrm{x}$ \\
\hline & $\mathrm{X}$ & $\mathrm{x}$ & $\mathrm{x}$ & $\mathrm{X}$ & $\mathrm{X}$ & $\mathrm{x}$ & $\mathrm{x}$ & $x$ \\
\hline & $\mathrm{x}$ & $\mathrm{x}$ & $\mathrm{x}$ & $\mathrm{x}$ & $\mathrm{x}$ & $\mathrm{X}$ & $\mathrm{x}$ & $\mathrm{x}$ \\
\hline & $\mathrm{X}$ & & & $\mathrm{X}$ & & $\mathrm{x}$ & & \\
\hline & $\mathrm{x}$ & $\mathrm{x}$ & & $\mathrm{x}$ & $\mathrm{X}$ & $\mathrm{x}$ & $\mathrm{x}$ & $x$ \\
\hline & $\mathrm{X}$ & $\mathrm{x}$ & & $\mathrm{X}$ & $\mathrm{X}$ & $\mathrm{x}$ & $\mathrm{x}$ & $x$ \\
\hline & $\mathrm{X}$ & $\mathrm{X}$ & & $\mathrm{X}$ & & $\mathrm{x}$ & $\mathrm{X}$ & $x$ \\
\hline & $\mathrm{x}$ & $\mathrm{x}$ & $\mathrm{X}$ & $\mathrm{x}$ & $\mathrm{X}$ & $\mathrm{X}$ & $\mathrm{x}$ & $\mathrm{x}$ \\
\hline & & & $\mathrm{X}$ & & $\mathrm{X}$ & & & \\
\hline & & & & & & & & \\
\hline
\end{tabular}

DSP, Echantillonnage en Début de Saison Pluvieuse FSP ; Echantillonnage en Fin de Saison Pluvieuse ; X, Présence d'une espèce. 
E. ILBOUDO -TAPSOBA et al. / Int. J. Biol. Chem. Sci. 5(2): 724-738, 2011

Tableau 4 : Richesse spécifique, équitabilité et indice de diversité par période dans les quatre (4) Micro Bassin-Versants (MBV) d'étude. Burkina Faso, 2006.

\begin{tabular}{lcccccccc}
\hline & \multicolumn{9}{c}{ Micro Bassin-Versants (MBV) } \\
\cline { 2 - 8 } & \multicolumn{2}{c}{ Kompienga } & \multicolumn{2}{c}{ Kouritenga } & \multicolumn{2}{c}{ Sanmatenga } & \multicolumn{2}{c}{ Soum } \\
Période & DSP & FSP & DSP & FSP & DSP & FSP & DSP & FSP \\
Richesse Spécifique & 34 & 30 & 18 & 21 & 20 & 24 & 41 & 32 \\
Indice de Shannon (H') & 1,6 & 1,8 & 1,5 & 1,6 & 1,2 & 1,8 & 2,3 & 2,3 \\
H' max & 5,1 & 4,9 & 4,2 & 4,4 & 4,3 & 4,6 & 5,4 & 5,0 \\
Equitabilité J' & 0,31 & 0,37 & 0,35 & 0,36 & 0,28 & 0,39 & 0,43 & 0,46 \\
\hline
\end{tabular}

DSP, Echantillonnage en Début de Saison Pluvieuse ; FSP, Echantillonnage en Fin de Saison Pluvieuse. 
E. ILBOUDO -TAPSOBA et al. / Int. J. Biol. Chem. Sci. 5(2): 724-738, 2011

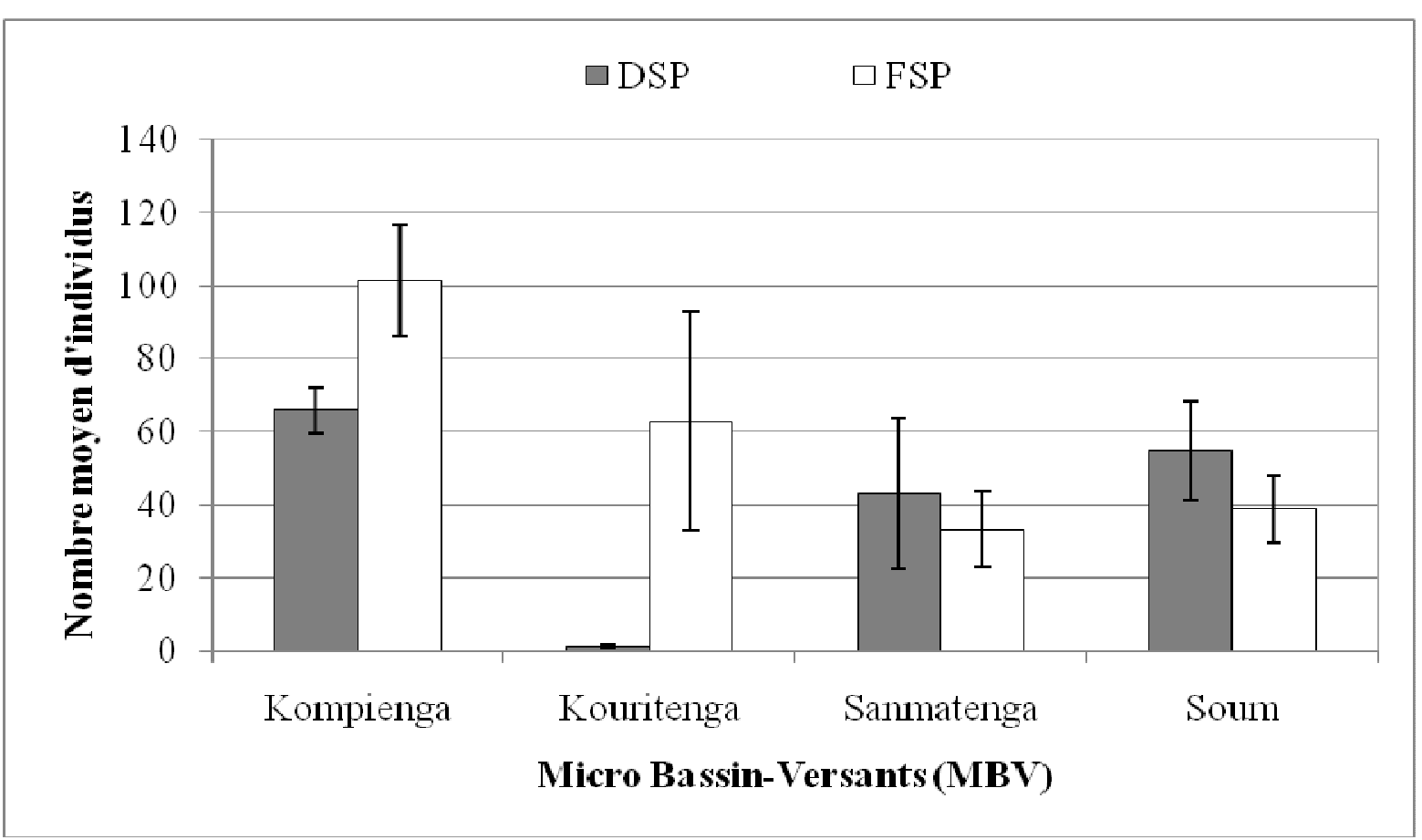

Figure 2 : Abondance relative moyenne des insectes (moyenne \pm écart type) par placette en fonction de la période de collecte et du Micro Bassin-Versant (MBV) d'étude. Burkina Faso, 2006.

DSP, Echantillonnage en Début de Saison Pluvieuse ; FSP, Echantillonnage en Fin de Saison Pluvieuse. 
E. ILBOUDO -TAPSOBA et al. / Int. J. Biol. Chem. Sci. 5(2): 724-738, 2011

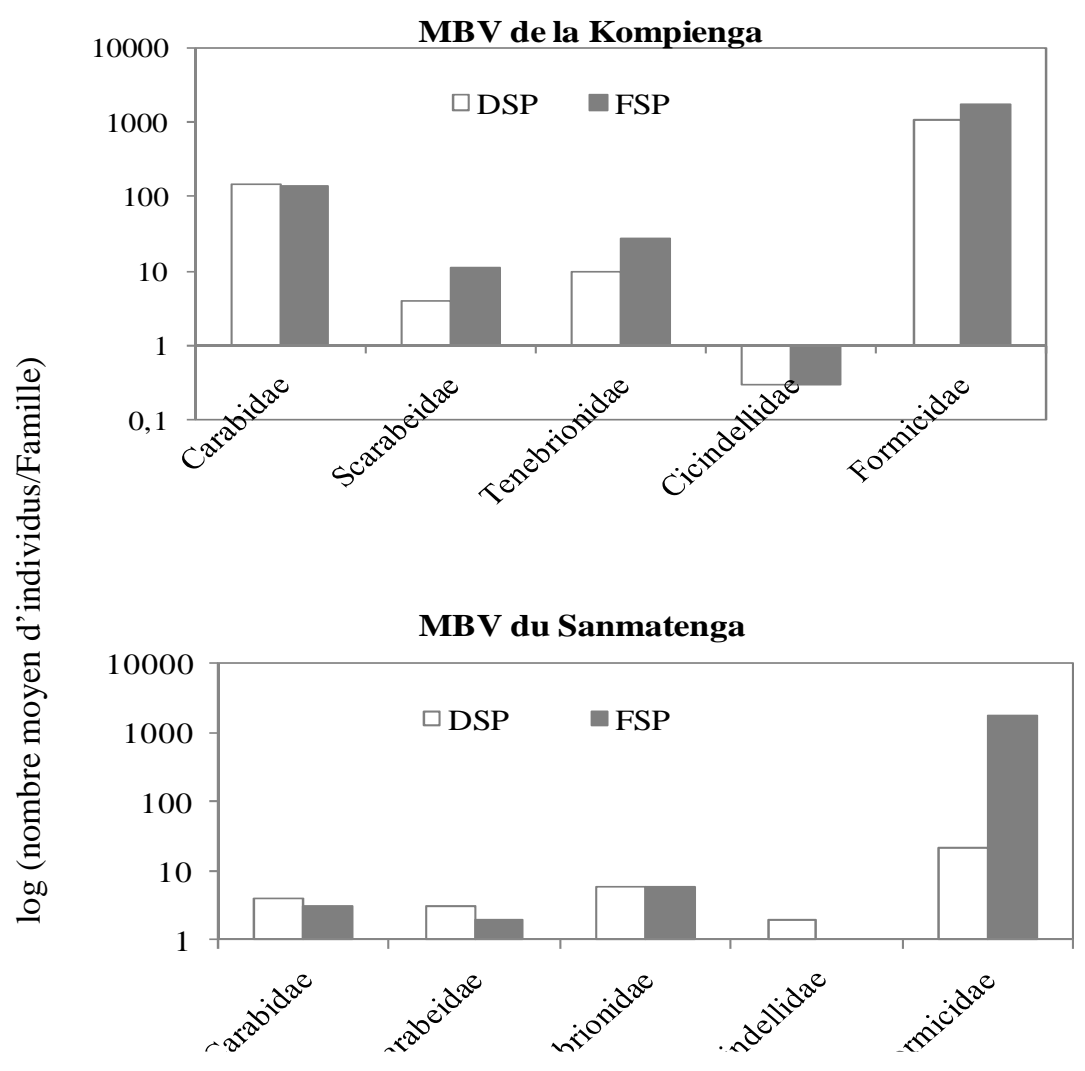

Principales familles d'insectes
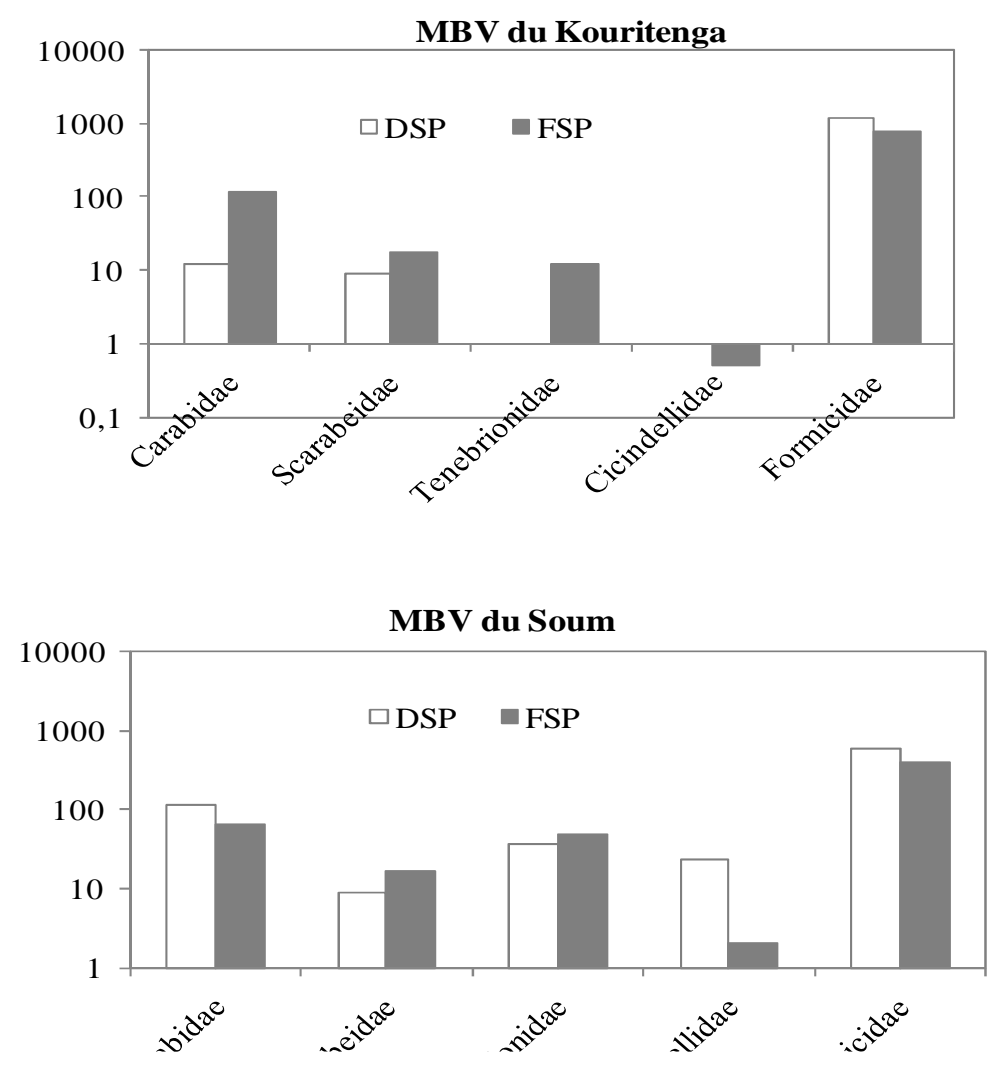

Principales familles d'insectes

Figure 3 : Abondance relative (log nombre moyen d'individus) des insectes dans les principales familles par placette en fonction de la période de collecte et du Micro Bassin-Versant (MBV) d'étude. Burkina Faso, 2006.

DSP, Echantillonnage en Début de Saison Pluvieuse FSP, Echantillonnage en Fin de Saison Pluvieuse. 
L'analyse de variance de KruskalWallis appliquée à l'indice de diversité de Shannon révèle que les sites d'échantillonnage n'ont pas eu d'influence sur la diversité au sein d'un même MBV (P>0,05) à l'exception de celui du Soum $\left(\mathrm{F}_{(56-11)}=36,87 ; \mathrm{P}<0,0001\right.$ et $\mathrm{F}_{(56-11)}=44,22 ; \mathrm{P}<0,0001$ respectivement pour la première et la seconde période d'échantillonnage).

Cependant, la période d'échantillonnage a influencé significativement l'indice J', en améliorant au cours de la seconde période la régularité des peuplements dans les $\mathrm{MBV}$ du soum $\left(\mathrm{F}_{(56-23)}=75,87 ; \quad \mathrm{P}<0,0001\right) \mathrm{du}$ Kouritenga $\left(\mathrm{F}_{(56-13)}=61,15 ; \mathrm{P}<0,0001\right)$ et de la Kompienga $\left(\mathrm{F}_{(56-21)}=33,12 ; \mathrm{P}<0,05\right)$. Cette influence de la période de collecte sur la régularité n'a toutefois pas été observée au Sanmatenga $\left(\mathrm{F}_{(56-13)}=9,92 ; \mathrm{P}=0,70\right)$.

\section{Abondance et représentativité des groupes entomologiques}

En moyenne, un nombre relativement important d'insectes (jusqu'à 100) a été collecté par placette. Cependant, l'abondance des insectes collectés varie suivant les zones d'étude et la période de collecte. Dans les MBV de la kompienga et du Kouritenga, le nombre d'insectes collectés augmente significativement du début à la fin de la saison de pluies (Fig. 1). Par contre, dans les MBV du Sanmatenga et du Soum, aucune différence significative n'a été observée entre les effectifs d'insectes en fonction de la période de collecte $(\mathrm{P}>0,05)$.

Deux familles, celles des Carabidae et des Formicidae, ont été plus abondantes que les autres et dominent donc les peuplements d'insectes dans les quatre (4) MBV (Fig. 2). Dans le MBV du Sanmatenga, les effectifs ont été très faibles au sein de toutes les familles à l'exception de celle des Formicidae. Le déséquilibre de répartition s'est accentué au sein du peuplement, dominé alors par la seule famille des Formicidae dans ce MBV (Fig. 2).

\section{DISCUSSION}

Cette étude donne des indications importantes, quoique préliminaires, de la diversité des peuplements d'insectes rampants au sol dans différents milieux du Burkina Faso. De façon générale, la richesse spécifique a été relativement faible dans les écosystèmes de bas-fonds qui ont comporté deux ordres majeurs, les Coléoptères et les Hyménoptères. Deux familles d'insectes, les Carabidae et les Formicidae, ont dominé les peuplements de tous les quatre (4) MBV, aussi bien par le nombre d'espèces que celui des individus les composant. Les espèces identifiées l'ont été sur la base de collections de références regroupant des spécimens de l'Afrique de l'Ouest. Aucune espèce nouvelle n'a encore été mise en évidence dans l'état actuel de nos investigations.

Les Carabidae sont des insectes très sensibles aux variations environnementales et sont reconnues pour leur qualité de bioindicateurs des écosystèmes naturels (Butterfield et al., 1995 ; Ings et Hartley, 1999) et agro-forestiers (Buchs, 2003 ; Venier et Pearce, 2006). De façon spécifique, l'humidité, la lumière et la température sont des facteurs importants qui influencent la distribution des Carabidae dans un habitat (Judas et al., 2002). Ainsi, les variations spatio-temporelles de la diversité de ces insectes au cours de cette étude sont à mettre en relation avec les conditions climatiques et écologiques qui prévalent dans la province où se situe le MBV d'étude. En effet, le MBV de la Kompienga, situé en zone plus arrosée, a été le plus riche en Carabidae. L'amélioration générale de la répartition des espèces au cours de la seconde période d'échantillonnage dans la plupart des MBV s'explique également par des conditions plus humides, des températures plus douces et un microclimat favorable engendrés par la saison pluvieuse.

La plus grande diversité observée au sein des Scarabaeidae, Cicindellidae et Tenebrionidae au Soum pourrait être liée à l'anthropisation poussée et de façon spécifique à l'activité économique dominante dans cette province du Burkina Faso. En effet, le Soum est surtout caractérisé par l'élevage de bovins et de petits ruminants. Ainsi, les animaux en s'abreuvant dans les bas fonds, y déposent de la bouse favorisant la diversité des insectes du sol en général et celle des Scarabaeidae en particulier (Fabre, 2000). Une telle observation a été réalisée lors d'études d'impact de l'anthropisation dans des aires protégées comme le parc du $\mathrm{W}$ au Burkina 
Faso (Samandoulgou, 2006) et au Benin (Sana, 2006).

La famille des Formicidae a été la seule à avoir montré une homogénéité de répartition dans les quatre (4) MBV. Cela suggère que les exigences écologiques de ce taxon sont requises dans tous les milieux étudiés. La présence de fourmis peut avoir des avantages écologiques. En effet, par leurs activités de construction et d'aménagement, ces insectes contribuent à aérer et à régénérer les sols, toute chose influençant la structure de la végétation (Lesica et Kannwoski, 1998). Il pourrait y avoir une corrélation entre les espèces de fourmis que nous avons rencontrées et les activités humaines menées dans chaque site respectif. Toutefois, les effets de perturbations de l'habitat sur les biocénoses de fourmis demeurent peu connus (Francœur, 2001), ce qui justifierait la poursuite d'investigations scientifiques dans cette direction.

\section{Conclusion}

Nos résultats suggèrent un approfondissement des études portant sur l'écologie des Carabidae, des Scarabaeidae et des Formicidae en vue de leur utilisation comme taxons indicateurs de l'état des écosystèmes de bas-fonds sous l'impact d'activités humaines. La détermination précise de la valeur écologique des espèces dominantes et de leur intérêt pour la gestion de ces milieux sera une étape importante vers cette réalisation. Les résultats obtenus permettent d'établir une situation de référence dans les MBV étudiés. Le suivi de l'évolution des taxa indicateurs identifiés à moyen ou long terme permettra donc d'évaluer l'impact des interventions menées dans les MBV étudiés sur leur viabilité écologique.

\section{REMERCIEMENTS}

Les auteurs expriment leur gratitude au projet de gestion des bas fonds du sahel au Burkina Faso (SILEM/PNGT2) qui, en collaboration avec la représentation de l'IUCN au Burkina Faso, a financé les recherches relatives à la biodiversité des arthropodes dans les écosystèmes de bas fonds dans quatre (4) provinces du Burkina Faso, et a octroyé une bourse doctorale à E. Ilboudo-
Tapsoba. Les remerciements s'adressent également aux responsables du muséum de l'IITA-Benin et du Laboratoire des Invertébrés Terrestres de l'IFAN-Sénégal pour leur assistance dans l'identification des espèces.

\section{REFERENCES}

Augusseau X, Liehoun E, Kara A. 2000. Evolution de l'organisation agraire dans deux terroirs d'accueil de migrants du Sud-Ouest du Burkina Faso : un même processus dans l'actuel front pionnier? In Forum National de la Recherche Scientifique et des Innovations Technologiques. CNRST (éd.): Ouagadougou, Burkina Faso.

Buchs W. 2003. Biodiversity and agrienvironmental indicators-general scopes and skills with special reference to the habitat level. Agricultur, Ecosystems \& Environment, 98: 35-78.

Butterfield J, Luff ML, Baines Veyre MD. 1995. Carabid beetle communities as indicators of conservation potential in upland forests. Forest Ecology and Management, 79: 63-77.

Delobel A, Tran M. 1993. Les Coléoptères des Denrées Alimentaires Entreposées dans les Régions Chaudes. ORSTOM, CTA : Paris, France ; 424.

Delvare G, Aberlenc H. 1999. Les Insectes d'Afrique et d'Amérique Tropicale. Clés pour la Recommandation des Familles. Imprimerie caballery-58500 Clamecy: France ;302.

Duelli P, Obrist MK. 2003. Biodiversity indicators: the choice of values and measures. Agriculture Ecosystems and Environment, 98: 87-98.

Fabre JH. 2000. Souvenirs Entomologiques: Etude sur l'Instinct et les Moeurs des Insectes (Tomes 1 et 2). Éd. Robert Laffont, coll. Bouquins.

Finnamore AT. 1996. The Advantages of Using Arthropods in Ecosystem Management. A Brief from the Biological Survey of Canada (Terrestrial Arthropods). 11 p.

Francœur A. 2001. Les fourmis de la forêt boréale du Québec (Hymenoptera: 
Formicidae). Le Naturaliste Canadien, 125(3): 108-114.

Fontès J, Guinko S. 1995. Carte de la végétation et de l'occupation des sols du Burkina Faso. Notice explicative, Ministère de la coopération française, Projet Campus, Toulouse, 68p.

Green RE, Cornell SJ, Scharlemann JPW, Balmford A. 2005. Farming and the fate of wild nature. Science, 307(5709): 550555.

Groombridge B, Jenkims MD. 2002. World Atlas of Biodiversity. Earth's Living Resources in the $21^{\text {st }}$ Century. California; 222-352.

Ings TC, Hartley SE. 1999. The effect of habitat structure on carabid communities during the regeneration of a native Scottish forest. Forest Ecology and Management, 119: 123-136.

Judas M, Dornieden K, Strothmann U. 2002. Distribution patterns of carabid beetle species at the landscape level. Journal of Biogeography, 29: 249 - 508.

Kremen C, Colwell Rk, Erwin TF, Murphy DD, Noss RF, Sanjayan MA. 1993. Terrestrial arthropod assemblage: their use in conservation planning. Convention Biology, 7(4): 796-808.

Lawton JH. 2001. All creatures great but mainly small. Ecol. Entomology, 26: 225 -226 .

Lesica P, Kannowski PB. 1998. Ants create hummocks and alter structure and vegetation of a montana fen. The American Midland Naturalist, 139: 5869.

Magurran AE. 2004. Measuring Biological Diversity. Blackwell Publishing: Malden, Oxford and Victoria; 256.

Mathieu P. 1994. Mouvements de population et transformations agricoles: le cas du Sud-Ouest du Burkina Faso. Cahiers $d u$ CIDEP, 20: 19-40.

New TR. 1999. Limits to species focusing in insect conservation. Annals of the Entomological Society of America, 92: $853-860$.

Powledge F. 2002. A look Back at the International Biodiversity observation Year. BioScience, 52: 1070 - 1079.
Ruxton GD, Beauchamp G. 2008. Some suggestions about appropriate use of the Kruskal-Wallis test. Animal Behaviour, 76: 1083-1087.

Samandoulgou Y. 2006. Identification d'insectes indicateurs de niveaux d'anthropisation des milieux dans le parc régional du $\mathrm{W}$ et sa périphérie. Composante du Burkina Faso. Mémoire de DEA, option production animale, Institue du développement rural (IDR), Université Polytechnique de BoboDioulasso, p. 41.

Samways MJ, Lockwood JA. 1998. Orthoptera conservation: Pests and paradoxes. Journal of Insect Conservation, 2: 143 - 149.

Sana Y. 2006. Identification d'insectes bioindicateurs dans le parc du W (Bénin) et sa périphérie. Mémoire de DEA, option production animale, Institut du Développement Rural (IDR), Université Polytechnique de Bobo-Dioulasso, p. 41.

Silem. 2005. Plan de Gestion Intégrée des Ecosystèmes du Micro Bassin-Versant Koulouko dans le Kouritenga. p. 47.

Silem. 2006a. Plan de Gestion Intégrée des Ecosystèmes du Micro Bassin-Versant du Béli (Province du Soum). p. 98.

Silem. 2006b. Plan de Gestion Intégrée des Ecosystèmes du Micro Bassin-Versant de Korsimoro dans la province du Sanmatenga. p. 87.

Silem. 2006c. Plan de Gestion Intégrée des Ecosystèmes du Micro Bassin-Versant du Lac de Barrage de la Kompienga. p. 151.

Venier LA, Pearce JL. 2006. The use of ground beetles (Coleoptera: Carabidae) and spiders (Araneae) as bioindicators of sustainable forest management: A review. Ecological Indicators, 6: 780-793.

Wiggins GB, Marshall SA, Downes JA. 1991. The importance of research collections of terrestrial arthropods. Bulletin of the Entomological society of Canada, 23(2): 16. 